\section{Mathematical Morphology Based Reference Signals Generation for Active Power Filters}

\section{A. Shintemirov}

In this paper the mathematical morphology theory is applied to implement the instantaneous power $p-q$ control strategy of a shunt active power filter. The simulation results indicate that the implemented control strategy is able to completely compensate the harmonic components in the load current and reactive power required by non-linear loads in the power system.

Introduction: Shunt active power filters (APFs) are one of the effective solutions to harmonic and reactive power compensation in power systems. Many APF control techniques have been proposed that require fast and exact estimation of line fundamental frequency and harmonic content of the current drawn by non-linear loads to ensure precise APF compensation. The instantaneous power $p-q$ theory is one of the widely applied and recognized approaches for generating reference current signal for APF control [1]. Recently, mathematical morphology (MM) has been successfully applied to power system signals processing due to its simple operation and robust performance [2,3]. In this paper an implementation of the $p-q$ theory based on a low-pass morphological filter is presented to reactive power and harmonics compensation with a shunt APF.

Reference Current Generation: Consider a 3-phase distribution line with a non-linear load, where the vectors of phase voltages and currents denote as $\left[\begin{array}{lll}u_{\mathrm{a}} & u_{\mathrm{b}} & u_{\mathrm{c}}\end{array}\right]^{\mathrm{T}}$ and $\left[\begin{array}{lll}i_{\mathrm{a}} & i_{\mathrm{b}} & i_{\mathrm{c}}\end{array}\right]^{\mathrm{T}}$ respectively.

According to the $p-q$ theory the voltages and currents can be transformed to stationary $\alpha-\beta-0$ coordinate system using the Clarke transformations as below [1]:

$$
\left[\begin{array}{l}
u_{\alpha} \\
u_{\beta} \\
u_{0}
\end{array}\right]=\mathbf{C}\left[\begin{array}{l}
u_{\mathrm{a}} \\
u_{\mathrm{b}} \\
u_{\mathrm{c}}
\end{array}\right] ; \quad\left[\begin{array}{c}
i_{\alpha} \\
i_{\beta} \\
i_{0}
\end{array}\right]=\mathbf{C}\left[\begin{array}{c}
i_{\mathrm{a}} \\
i_{\mathrm{b}} \\
i_{\mathrm{c}}
\end{array}\right] .
$$

The inverse transformations are defined as follows:

$$
\left[\begin{array}{c}
u_{\mathrm{a}} \\
u_{\mathrm{b}} \\
u_{\mathrm{c}}
\end{array}\right]=\mathbf{C}^{-1}\left[\begin{array}{l}
u_{\alpha} \\
u_{\beta} \\
u_{0}
\end{array}\right] ; \quad\left[\begin{array}{c}
i_{\mathrm{a}} \\
i_{\mathrm{b}} \\
i_{\mathrm{c}}
\end{array}\right]=\mathbf{C}^{-1}\left[\begin{array}{c}
i_{\alpha} \\
i_{\beta} \\
i_{0}
\end{array}\right] .
$$

Considering three-phase three wire power system, the three-phase instantaneous active and imaginary reactive powers are defined in the stationary system as follows:

$$
\left[\begin{array}{l}
p \\
q
\end{array}\right]=\left[\begin{array}{cc}
u_{\alpha} & u_{\beta} \\
u_{\beta} & -u_{\alpha}
\end{array}\right]\left[\begin{array}{l}
i_{\alpha} \\
i_{\beta}
\end{array}\right] .
$$

Each power component in (3) can be separated into a mean value or DC component and an oscillating part or AC component:

$$
p=\bar{p}+\tilde{p} \quad \text { and } \quad q=\bar{q}+\tilde{q}
$$

The DC components $\bar{p}$ and $\bar{q}$ correspond to the conventional three-phase active and reactive powers and the remaining $\mathrm{AC}$ component $\tilde{p}$ and $\tilde{q}$ are mainly related to the presence of harmonics in the load currents [1].

According to the constant power compensation control strategy based on the $p-q$ theory in order to draw a constant instantaneous power from the source, the shunt active filter should be installed as close as possible to the non-linear load, and should compensate the oscillating active power component $\tilde{p}$ of this load. Additionally, the APF should compensate the total imaginary power $q$ of the load [1].

To calculate reference currents corresponding to the powers $\tilde{p}$ and $q$, at first, the DC component of the active power $\bar{p}$ is extracted using a low-pass filter. Then, the currents corresponding to the three-phase active powers are obtained as follows:

$$
\begin{gathered}
{\left[\begin{array}{c}
i_{\alpha \bar{p}} \\
i_{\beta \bar{p}}
\end{array}\right]=\frac{1}{u_{\alpha}^{2}+u_{\beta}^{2}}\left[\begin{array}{cc}
u_{\alpha} & u_{\beta} \\
u_{\beta} & -u_{\alpha}
\end{array}\right]\left[\begin{array}{c}
\bar{p} \\
0
\end{array}\right] ;} \\
{\left[\begin{array}{c}
i_{\mathrm{a} \bar{p}} \\
i_{\mathrm{b} \bar{p}} \\
i_{\mathrm{c} \bar{p}}
\end{array}\right]=\mathbf{C}^{-1}\left[\begin{array}{c}
i_{\alpha \bar{p}} \\
i_{\beta \bar{p}} \\
0
\end{array}\right] .}
\end{gathered}
$$

The reference currents are then calculated by subtracting the currents $\left[\begin{array}{lll}i_{\mathrm{a} \bar{p}} & i_{\mathrm{b} \bar{p}} & i_{\mathrm{c} \bar{p}}\end{array}\right]^{\mathrm{T}}$ from the load currents $\left[\begin{array}{lll}i_{\mathrm{a} \mathrm{L}} & i_{\mathrm{bL}} & i_{\mathrm{c} \mathrm{L}}\end{array}\right]^{\mathrm{T}}$ :

$$
\left[\begin{array}{c}
i_{\text {a ref }} \\
i_{\mathrm{b} \text { ref }} \\
i_{\mathrm{c} \mathrm{ref}}
\end{array}\right]=\left[\begin{array}{c}
i_{\mathrm{a} \mathrm{L}} \\
i_{\mathrm{b} \mathrm{L}} \\
i_{\mathrm{c} \mathrm{L}}
\end{array}\right]-\left[\begin{array}{c}
i_{\mathrm{a} \bar{p}} \\
i_{\mathrm{b} \bar{p}} \\
i_{\mathrm{c} \bar{p}}
\end{array}\right] .
$$

A Low-Pass Morphological Filter: Mathematical morphology is based on the set theory and extracts the object features by processing signals using a a structuring element (SE). The SE, which can be considered as a moving window, slides through the signal, interacts with the samples covered by the window, and detects specific features in the signal [4].

Dilation $\oplus$ and erosion $\ominus$ are the two basic morphological operators. The dilation and erosion of a signal $f(t)$ by a SE $g(s)$, are defined as follows, respectively [2]:

$$
\begin{aligned}
& (f \oplus g)(t))=\max _{\tau}\{f(t-\tau)+g(\tau)\} ; \\
& (f \ominus g)(t))=\min _{\tau}\{f(t+\tau)-g(\tau)\},
\end{aligned}
$$

The opening $\circ$ and closing $\bullet$ morphological operators are used to remove sharp peaks and valleys in a signal, respectively, and are defined as below [5]:

$$
\begin{aligned}
& (f \circ g)=(f \ominus g) \oplus g ; \\
& (f \bullet g)=(f \oplus g) \ominus g .
\end{aligned}
$$

There are two well-known alternative sequential filters, i.e. the openclosing filter and close-opening filter, which are the cascade connections of the opening and closing operators in different order. These filters were designed to remove impulsive noise [5] and can be used for high-frequency oscillations removal in signals, thereby, acting as low-pass filters. In order to lower the output deviation and achieve the best performance, the two filters can be cascaded to form a combined low-pass filter, the average of open-closing and close-opening operations [3]:

$$
y=\frac{(f \circ g) \bullet g+(f \bullet g) \circ g}{2},
$$

where $y$ and $f$ are the output and input signals, respectively, and $g$ is the SE.

Simulation and Results: The effectiveness of the proposed reference signal generation based on the low-pass morphological filter for APF operation is simulated using the MATLAB/Simulink environment. A standard IGBT inverter block with a series inductor on the $\mathrm{AC}$ side and a DC capacitor models an APF in a 3-phase power system with a non-linear load. The APF control system is represented by the three blocks: morphological-filter-pq, reference current generation and hysteresis PWM.

The morphological-filter-pq block contains blocks to convert voltage and current signals from $a-b-c$ to $\alpha-\beta-0$ coordinate systems according to (1)-(2), a block for calculation instantaneous active power $p$ according to (3), a low-pass block for extraction of $\bar{p}$ from $p$, and blocks for calculation currents $i_{\alpha \bar{p}}, i_{\beta \bar{p}}$ and $i_{\mathrm{a} \bar{p}}, i_{\mathrm{b} \bar{p}}, i_{\mathrm{c} \bar{p}}$ using (5) and (6).

The low-pass block realizes the morphological filter described in the previous section. It contains the the opening and closing blocks applying a flat SE with its origin at the centre. The length of SE is quarter of a wavelength at the fundamental frequency, $5 \mathrm{~ms}$, chosen to eliminate the higher frequency components from the input signal. Applying this SE directly will cause at least a quarter-cycle delay, since the extraction process require the quarter of a cycle of a current waveform data stored in buffer blocks. The reference current generation block realizes equation (7). The hysteresis PWM to generate a control pulses for the IGBT bridge is chosen due its simplicity and fastness.

The results of the simulation are presented in Fig. 1. The non-linear load in the simulated power system model is represented by two diode rectifiers which are phase-shifted by 30 degrees. The second rectifier is connected after 20 cycles to change the load from 6-pulse to 12-pulse.

At the start of simulation $(t=0 \mathrm{~s})$ only one diode rectifier is in circuit. The DC current of the load rectifier is set at 2000A. The source and the load currents are the same. After 1 cycle at $t=0.02 \mathrm{~s}$ the active filter is on. It takes about half of a cycle for the APF to generate the reference current and compensate the source current. Analysis of the load and source phase A current on the 3 rd cycle, it was found out the APF has effectively reduced the the total harmonic distortion (THD) from $23.13 \%$ on load side to $2.7 \%$ on source side. 

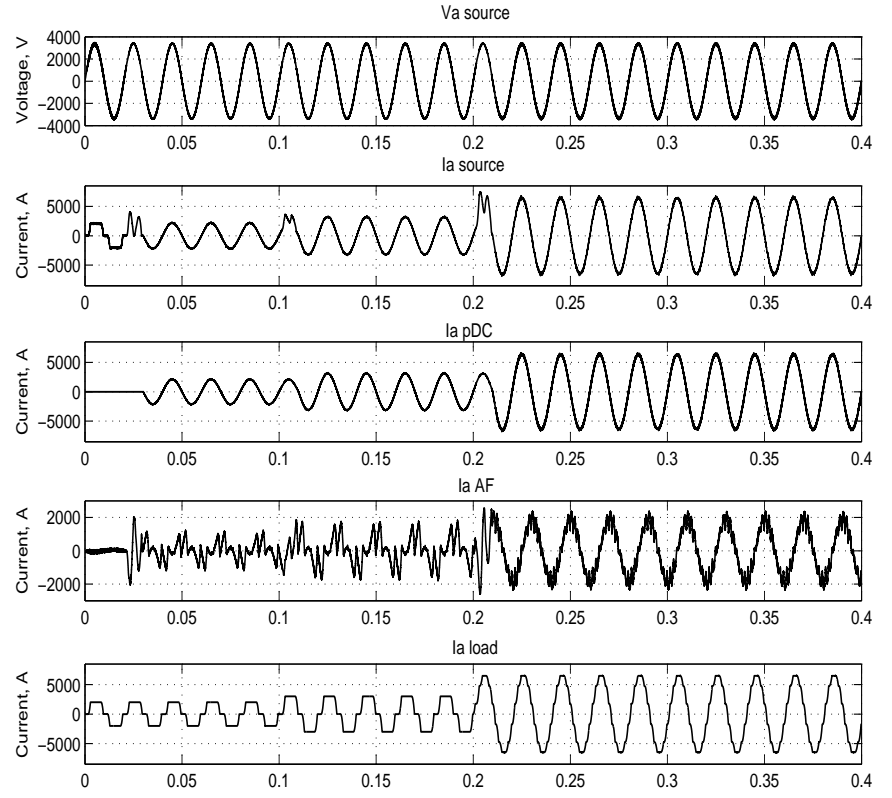
$\mathrm{Vdc}$

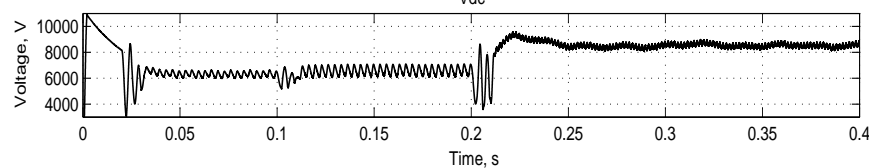

Fig. 1. Performance of active power filter (phase A signals).

At $t=0.1 \mathrm{~s}$, the DC current of the load rectifier is increased from 2000A to $3000 \mathrm{~A}$. The APF has effectively responded to this change in load and captured the new reference current within half of a cycle. At $t=0.2 \mathrm{~s}$, the second diode rectifier is connected, thus producing a 12-pulse load. It is seen that the APF again captures the new reference current within half of a cycle. The analysis of the load and source phase A current on the 13th cycle shows that the APF has effectively reduced the THD from 5.6\% to $1.82 \%$.

Figure 2 illustrates the comparison of the mean active and reactive powers on the source and load sides. It is seen that the APF has completely compensated the reactive power on the source side. A comparison of the phase A source and load currents with the phase A voltage showed that the APF eliminated the phase shift between the phase A voltage and current on the source side.

Conclusion: In this paper the morphological filtering technique is applied to implement an active power filter control based on the instantaneous power $p-q$ theory. The developed computer-aided simulation model in the MATLAB/Simulink is applied for studying the detailed behavior of
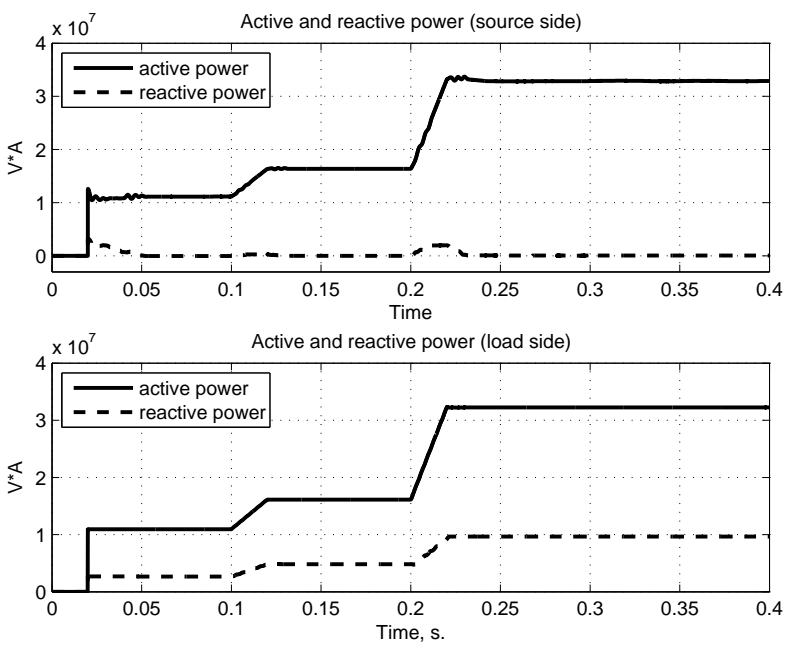

Fig. 2. Mean active and reactive power on the source and load sides. the system for harmonic and reactive power compensation. The simulation results indicate that the implemented control strategy is able to completely compensate the harmonic components of the supply current well below 5\% limits, required in the IEEE-519 standard, and reactive power required by the non-linear loads in the power system.

In order to improve the harmonic compensation and stability of the APF control system, a feedback control of the APF DC capacitor voltage needs to be introduced. This will be done during practical implementation of an APF prototype for further experimental study of the proposed control strategy utilizing MM algorithms.

\section{A. Shintemirov (Nazarbayev University, Astana, Kazakhstan)}

E-mail: ashintemirov@nu.edu.kz

\section{References}

1 Akagi, H. and Watanabe, E. H. and Aredes, M.: Instantenious Power Theory and Applications to Power Conditioning, John Wiley \& Sons, Inc., 2007

$2 \mathrm{Lu}, \mathrm{Z}$. and Ji, T. Y. and Wu, Q. H.: 'Morphological Transform for Removal of Exponentially Decaying DC-Offset', Electronics Letters, 2008, 44(9), pp. 595-596

3 Lu, Z. and Wu, Q. H. and Fitch, J.: 'A Morphological Filter for Estimation of Power System Harmonics', Proceedings of the IEEE 2006 International Conference on Power System Technology, 2006, pp. 1-5

4 Gonzalez, R. C. and Woods, R. E.: Digital Image Processing. Third Edition, Prentice Hall, 2007

5 Maragos, P. and Schafer, R. W.: 'Morphological Systems for Multidimensional Signal Processing', Proceedings of the IEEE, 1990, 78(4), pp.690-710 